

\title{
Frequency-modulation spectroscopy with blue diode lasers
}

\author{
Ulf Gustafsson, Gabriel Somesfalean, Janis Alnis, and Sune Svanberg
}

\begin{abstract}
Frequency-modulation spectroscopy provides ultrasensitive absorption measurements. The technique is especially adaptable to diode lasers, which can be modulated easily, and has been used extensively in the near-infrared and infrared spectral regions. The availability of blue diode lasers now means that the accessible wavelength region can be increased. We successfully demonstrate wavelength-modulation spectroscopy and two-tone frequency-modulation spectroscopy for the weak second resonance line of potassium at $404.8 \mathrm{~nm}$ and for the transition at $405.8 \mathrm{~nm}$ in lead, starting from the thermally populated $6 p^{2}{ }^{3} P_{2}$ metastable level. Information on the modulation parameters is obtained with a fitting procedure. Experimental signal-to-noise ratios at different absorption levels are compared with theoretical signal-to-noise ratios and show good agreement. Detection sensitivities of $2 \times 10^{-6}$ and $5 \times 10^{-6}$ for wavelength and two-tone frequency-modulation spectroscopy, respectively, for a $120-\mathrm{Hz}$ bandwidth are demonstrated. (C) 2000 Optical Society of America

OCIS codes: $300.6260,300.6380,300.1030$.
\end{abstract}

\section{Introduction}

Diode laser absorption spectroscopy based on frequency modulation (FM) is a commonly used method for fast and ultrasensitive detection of minute concentrations of gas. ${ }^{1-6}$ The interaction between a modulated laser field and an absorbing sample leads to the generation of an absorption-related signal that can be detected at the applied modulation, at an overtone, or at an intermediate frequency by use of frequency- and phase-sensitive electronics. Characteristically this process shifts the detection band to a high-frequency region, where the laser excess $(1 / f)$ noise is avoided. Although a variety of FM methods have been implemented, they actually represent limiting cases of the same technique. Depending on the number of modulation tones, on the choice of modulation frequency relative to the spectral width of the absorbing feature, and on the detection frequency, the methods are referred to as wavelengthmodulation spectroscopy (WMS), 7 single-tone FM

The authors are with the Department of Physics, Lund Institute of Technology, P.O. Box 118, SE-221 00 Lund, Sweden. J. Alnis is on leave from the Institute of Atomic Physics and Spectroscopy, University of Latvia, Rainis Boulevard 19, LV-1586 Riga, Latvia. The e-mail address for S. Svanberg is sune.svanberg@fysik.lth.se.

Received 28 October 1999; revised manuscript received 21 April 2000.

0003-6935/00/213774-07\$15.00/0

(C) 2000 Optical Society of America spectroscopy (STFMS), ${ }^{8}$ or two-tone FM spectroscopy (TTFMS). 9

In WMS the modulation frequency is much smaller than the half-width of the absorbing feature, whereas STFMS and TTFMS are characterized by modulation frequencies that are comparable to or larger than the half-width of the absorbing feature. WMS is usually performed at kilohertz frequencies (low-frequency WMS), with conventional lock-in amplifiers used for signal detection. For maximum sensitivity a large FM index, defined as the ratio between the maximum frequency deviation and the modulation frequency, is required. The sensitivity can be significantly improved by use of megahertz modulation frequencies (high-frequency WMS) and harmonic detection at moderate FM indices. ${ }^{10-12}$ Inasmuch as STFMS and TTFMS use high modulation frequencies, high sensitivity can be achieved with a low FM index. ${ }^{10-12}$ STFMS used for broad atmospheric-pressure lines with linewidths of some gigahertz requires detection electronics with matching bandwidths. Such instruments are complicated, expensive, and not always available in the wavelength region of interest. TTFMS circumvents this problem by using high FM of the laser at two closely spaced frequencies and detecting the signal at the difference frequency, often in the megahertz range. Thus, in TTFMS it is possible to use detection electronics of moderate bandwidth and still preserve high sensitivity at a low FM index. In the high-FM techniques (high-frequency WMS, STFMS, and TTFMS), the lock-in amplifier is 
replaced by discrete components. The use of FM at high frequencies offers the possibility of quantumnoise-limited detection. ${ }^{13-17}$

Diode lasers are especially well suited for highsensitivity absorption spectroscopy because one can both tune their emission wavelengths over the whole absorption profile by changing the temperature of the laser capsule and modulate their frequencies directly by applying an ac current on the drive current. Recently the Nichia Corporation introduced blue cw GaN diode lasers that emit near $400 \mathrm{~nm} .{ }^{18}$ Gustafsson et $a l .{ }^{19}$ recently demonstrated the usefulness of these lasers for spectroscopy by performing experiments with potassium vapor. No attempts at sensitive detection were made in these experiments, which employed direct absorption, laser-induced fluorescence, and optogalvanic spectroscopy. Here we extend this study by using the blue diode laser and perform WMS and TTFMS on potassium as well as on lead. We study the second resonance line, $4 s^{2} S_{1 / 2}-5 p{ }^{2} P_{3 / 2}$, in potassium $\left({ }^{39} \mathrm{~K}\right)$ at $404.8 \mathrm{~nm}$. We also record the transition in lead $\left({ }^{208} \mathrm{~Pb}\right)$ at $405.8 \mathrm{~nm}$, starting from the thermally weakly populated $6 p^{2}{ }^{3} P_{2}$ metastable level situated $1.3 \mathrm{eV}$ above the ground state. Absorption signals are measured at different temperatures and thus at different absorptions. By means of a fitting procedure, information regarding the modulation parameters is obtained. We examine the possibilities of making highly sensitive absorption measurements in what is for diode lasers a new and interesting wavelength region and compare experimentally deduced and theoretically calculated signal-to-noise ratios (SNR's). To achieve high sensitivity for both WMS and TTFMS, we perform WMS in the high-frequency wavelength-modulation regime, applying a modulation frequency of $5 \mathrm{MHz}$ and detecting the second harmonic at $10 \mathrm{MHz}$, whereas for TTFMS we use modulation frequencies near $900 \mathrm{MHz}$, i.e., larger than the Doppler widths (HWHM), which are less than 800 $\mathrm{MHz}$ for the transitions studied and detect beat signals at the same frequency as for WMS.

\section{Experiment}

A schematic of the setup for WMS and TTFMS experiments is shown in Fig. 1. The blue diode laser (Nichia NLHV500) has a nominal wavelength of 404 $\mathrm{nm}$ at $25{ }^{\circ} \mathrm{C}$ and a typical output power of $5 \mathrm{~mW}$. The laser diode is placed in a thermoelectrically cooled mount and is current and temperature controlled by a precision diode laser driver (Melles Griot 06DLD103). The diverging laser beam is collimated by a molded glass aspheric lens (Geltech C230TM-A) and is divided by a neutral-density filter. One part of the beam is transmitted through a small electrically heated oven into which either a 4-cm-long potassium cell or a 3.5-cm-long lead cell is placed. The other part of the beam is directed through a lowfinesse confocal Fabry-Perot etalon (free spectral range, $1.5 \mathrm{GHz}$ ) for frequency calibration of the absorption spectrum. Both beams are focused on detectors that contain $\mathrm{p}-\mathrm{i}-\mathrm{n}$ photodiodes (Hamamatsu S-1190) and homemade transimpedance amplifiers.

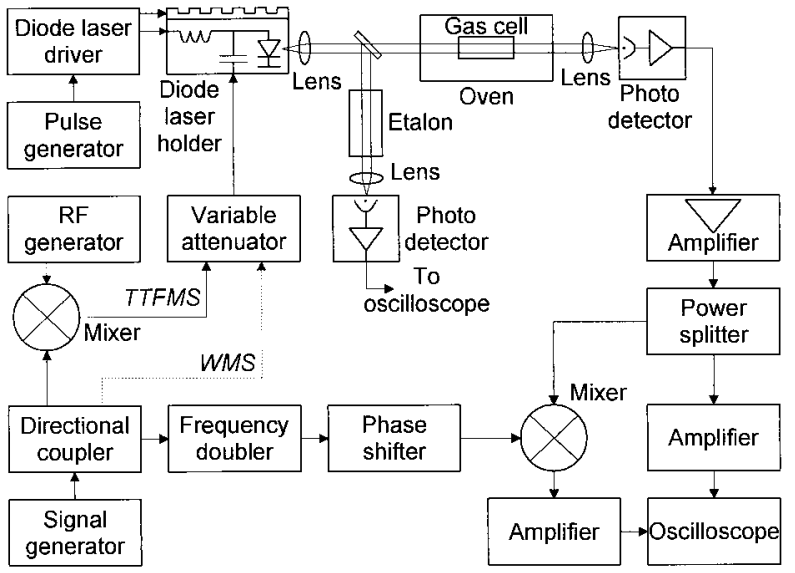

Fig. 1. Experimental setup for direct absorption spectroscopy, WMS, and TTFMS of potassium and lead vapor in sealed-off cells.

Wavelength scanning is achieved by repetitive application of a rectangular current pulse (Tektronix RG501) with a duration of $1-2 \mathrm{~ms}$ and a repetition rate of $100 \mathrm{~Hz}$ to the diode laser drive current, which is biased below the threshold current. A heatinduced constant-current wavelength shift is produced during the rectangular current pulse if the rise time of the pulse is short compared with the growth rate of the junction temperature. This wavelengthscanning technique minimizes variations in laser intensity and spectral characteristics and ensures an almost constant FM index during the scan. ${ }^{20,21}$ It also permits simultaneous recording of the absorption line and of the zero intensity level in direct $a b$ sorption. The free-running laser typically lases on a few modes separated by $\sim 0.05 \mathrm{~nm}$, as evidenced in a separate test with a high-resolution spectrometer. However, judicious choices of temperature and drive current ensure nearly single-mode operation.

The FM schemes for WMS and TTFMS are almost identical; the only difference is in the method of generating the modulation frequencies. In TTFMS we generate the two modulation frequencies by mixing a $905-\mathrm{MHz}$ signal (Wavetek 2510A) and a $5.35-\mathrm{MHz}$ signal (Tektronix SG503) in a frequency mixer (MiniCircuits ZFM-4H). For WMS, only the $5.35-\mathrm{MHz}$ modulation frequency is used. The modulation current, before it is superimposed upon the laser drive current, passes a variable attenuator, which allows the radio-frequency (rf) power to the diode laser to be varied. We detect the TTFMS and WMS signals by mixing (Mini-Circuits ZFM-3) the amplified (MiniCircuits ZFL-1000LN) beat and second-harmonic signals (at $10.7 \mathrm{MHz}$ ) from the detector with the frequency-doubled (Mini-Circuits FD-2) and appropriately phase shifted (Synergy PP-921) $5.35-\mathrm{MHz}$ signal. We adjust the rf power for the highest possible WMS and TTFMS signal amplitudes that can be achieved without the introduction of any significant modulation broadening. We split off (Mini-Circuits ZFRSC-2050) one part of the detector signal before the frequency mixer so we can observe the direct 
absorption signal. Where appropriate, bandpass-, high-pass, and low-pass filters are inserted to prevent the modulation frequencies from reaching the demodulation mixer, the detection frequency signal from reflecting back toward the laser, and the modulated absorption-related signals from influencing the direct absorption signals. The direct and demodulated signals are amplified and low-pass filtered at $30 \mathrm{kHz}$ in low-noise preamplifiers (Stanford SR560) and then averaged 256 times in a digital oscilloscope (Tektronix TDS520B). Thus the effective bandwidth of the detection system is approximately $120 \mathrm{~Hz}$. Finally, the recorded waveforms are transferred to a computer for processing and evaluation.

In WMS it is possible to record both the absorption and the dispersion related to the probed medium. Here we consider only the absorption-related signal by making the appropriate choice of detection phase. We can do this because the in-phase (0 or $\pi$ ) component of the signal corresponds to pure absorption, whereas the quadrature $( \pm \pi / 2)$ component corresponds to pure dispersion. In TTFMS a small part of the dispersion component will fall into the detection angle, provided that the detection phase (approximately 0 or $\pi$ ) is adjusted for optimum signal amplitude. ${ }^{22}$ However, for the transitions studied, the chosen modulation frequencies, and the precision of the detection phase adjustment $( \pm 5 \%)$, the dispersion component is less than $0.1 \%$ of the absorption component and is thus negligible.

\section{Measurements}

Natural potassium consists of two isotopes, ${ }^{39} \mathrm{~K}(93 \%)$ and ${ }^{41} \mathrm{~K}(7 \%)$, both with a nuclear spin of $3 / 2$. The studied sealed-off potassium gas cell contains only the isotope ${ }^{39} \mathrm{~K}$. We note that the existence of a nonzero nuclear spin for potassium gives rise to a hyperfine splitting of the $4 s^{2} S_{1 / 2}-5 p^{2} P_{3 / 2}$ transition. However, because of the small magnetic moment of the potassium nucleus, the hyperfine splittings are small; for ${ }^{39} \mathrm{~K}$ the ground-state splitting is only 462 $\mathrm{MHz}$ and the upper-state splitting is $\sim 2$ orders of magnitude smaller. ${ }^{23}$ Thus the Doppler-broadened potassium line, with a half-width of $\sim 800 \mathrm{MHz}$ for the temperatures used here, is not expected to show any structure.

Typical recorded spectra for direct absorption and TTFMS measurements at $90^{\circ} \mathrm{C}$ in potassium are shown in Fig. 2, where the low-finesse etalon fringes are also displayed. The WMS line shape is almost identical to that of TTFMS and is not shown. Because a rectangular current pulse is used for wavelength scanning, the laser output power is almost constant during the scan, the zero intensity level can be seen in the direct absorption recording, and the frequency sweep is quite nonlinear. This means that neither any laser output power rectification nor any separate zero intensity recording is necessary with direct absorption. It means, though, that the frequency scale has to be linearized, and subsequently this was done for all the spectra shown in the remaining part of this paper.

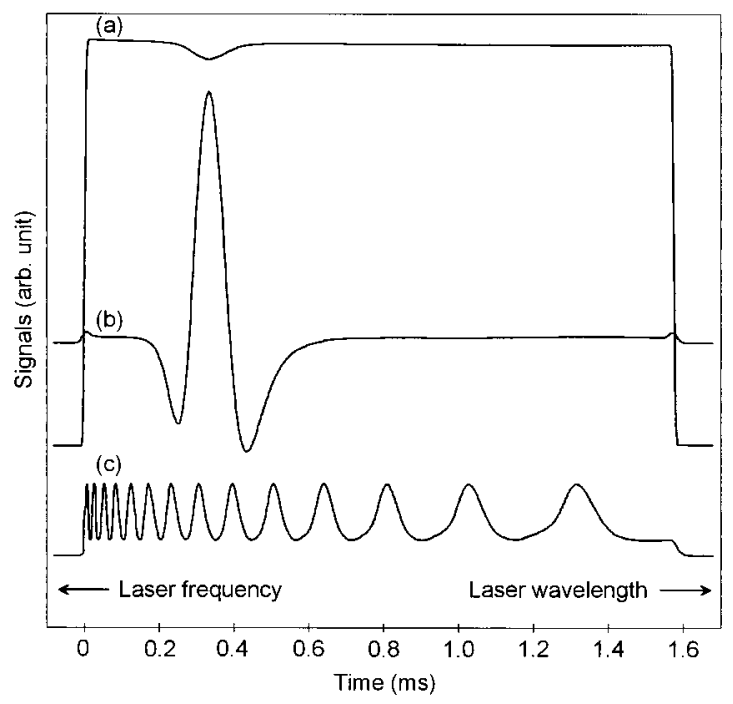

Fig. 2. (a) Direct absorption and (b) TTFMS spectra of the $4 \mathrm{~s}$ ${ }^{2} S_{1 / 2}-5 p{ }^{2} P_{3 / 2}$ line at $404.8 \mathrm{~nm}$ recorded with a rectangular current pulse. (c) Corresponding recording of the Fabry-Perot etalon fringes.

WMS and TTFMS line shapes for potassium vapor were recorded from $\sim 90{ }^{\circ} \mathrm{C}$ to room temperature. In Fig. 3, linearized recordings at 60 and $30^{\circ} \mathrm{C}$, corresponding to peak absorptions of $1.6 \times 10^{-3}$ and $6.7 \times$ $10^{-5}$, respectively, are displayed. Interference fringes, caused by reflections between the neutraldensity filter and the detector surface, are clearly visible in the recording of the TTFMS line shape at $30{ }^{\circ} \mathrm{C}$. Although FM spectroscopy offers the possibility of quantum-noise-limited detection, the minimum achievable sensitivity is in practice often set by

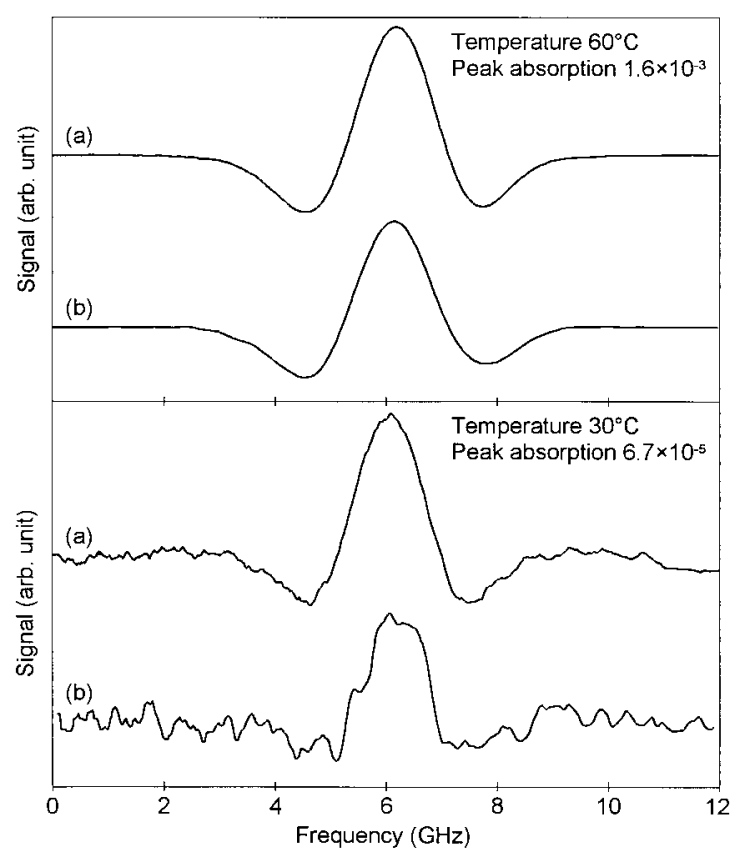

Fig. 3. Linearized (a) WMS and (b) TTFMS recordings for potassium at two cell temperatures. 


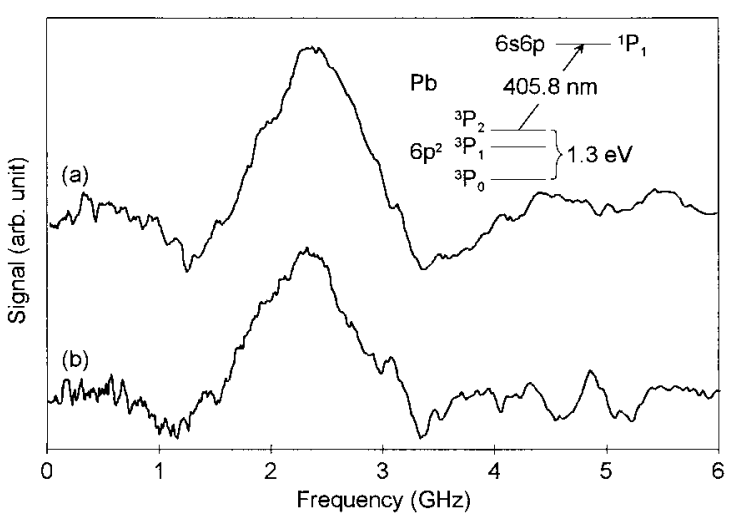

Fig. 4. Linearized (a) WMS and (b) TTFMS recordings of the lead 405.8-nm line. Inset, diagram of the structure of lead.

interference fringes that are seen as a periodically oscillating background and originate from spurious reflections along the laser beam path. The interference effect can often be removed or largely reduced by careful angling of all transmissive optics, and, as can be seen from the WMS recording in Fig. 3 [upper curve (a)], a slight adjustment of the neutral-density filter removes the interference fringes.

It is necessary to perform experiments at considerably higher temperatures if one wishes to observe the absorption in lead, because of the low vapor pressure of this metal. We studied the transition at $405.8 \mathrm{~nm}$, starting from the thermally weakly populated $6 p^{2}{ }^{3} P_{2}$ metastable level situated $1.3 \mathrm{eV}$ above the ground state. The lead experiments were performed at temperatures that ranged from 500 to $700{ }^{\circ} \mathrm{C}$, corresponding to ground-state atomic densities of $2 \times 10^{17} \mathrm{~m}^{-3}$ to $7 \times 10^{19} \mathrm{~m}^{-3}$ and to Boltzmann factors of $2 \times 10^{-8}$ to $9 \times 10^{-7}$. These atomic densities can be compared with those in the potassium cell, which range from $3 \times 10^{14} \mathrm{~m}^{-3}\left(20^{\circ} \mathrm{C}\right)$ to $3 \times$ $10^{17} \mathrm{~m}^{-3}\left(90^{\circ} \mathrm{C}\right)$. The lead gas cell contains a natural mixture of lead isotopes, but the specific measurements were performed on the transition corresponding to the ${ }^{208} \mathrm{~Pb}$ isotope with a nuclear spin of 0 . The isotope, which has no hyperfine splitting because of the zero nuclear spin, has a Doppler width of $\sim 550 \mathrm{MHz}$.

The linearized WMS and TTFMS recordings at $600{ }^{\circ} \mathrm{C}$ (peak absorption, $2.0 \times 10^{-4}$ ) are shown in Fig. 4. These recordings were also influenced by interference fringes that originated from a reflection from the detector surface back into the diode laser cavity. Despite cautious angling of all optics as well as of the diode laser and the detector, we were not able to suppress completely the interference fringes in the lead experiments. We explain the difference in the results of the experiment with potassium from that with lead as being due to different diode laser behaviors at the two wavelengths because different current and temperature settings were used. Another unwanted and limiting effect in the lead experiments is a diode laser mode jump close to the transition on the low-frequency side. The frequency position of the mode jump varies in time, and this variation adds an irregular background to the recorded line shapes.

\section{Results and Discussion}

Here we first determine the modulation parameters in WMS and TTFMS by means of a fitting procedure of the recorded line shapes. Then we use these modulation parameters to calculate the theoretical SNR's and compare them with the experimentally deduced SNR's. Finally, we estimate the maximum achievable sensitivity of this blue diode laser-based spectrometer. The evaluation was performed mainly with the experimental data for potassium, because, as we noted above, the lead experiments were hampered by interference fringes and a diode laser mode jump, in which case, the SNR was set by these effects rather than by the fundamental noise sources in a FM spectrometer. The formalism that describes the FM theory, including calculations of WMS and TTFMS line shapes and SNR's, can be found, e.g., in Refs. 22 and 24-26.

FM of diode lasers is always accompanied by residual amplitude modulation (RAM), which is noticeable in WMS and TTFMS recordings as a line-shape asymmetry. This RAM, which is accounted for in the FM theory by the AM index $M$ and the AM-FM phase difference $\psi$, is an effect that is caused by the laser intensity not being exactly uniform over the FM range, and it is undesirable because it carries some of the low-frequency noise into the FM signal. The AM index and the AM-FM phase difference are closely connected in theory and are difficult to determine independently. A value of $\psi=\pi / 2$ for the AM-FM phase difference is generally considered to be a good approximation for diode lasers, ${ }^{22,27,28}$ and, in what follows, we adopt this value.

As we mentioned above, the studied transition in potassium actually consists of several hyperfine components. These can be divided mainly into two Gaussians that have a half-width given by the Doppler width, are separated by $462 \mathrm{MHz}$ and have an intensity ratio of $5 / 3$, which is equal to the statistical weights of the two hyperfine ground-state levels $(F=$ 2 and $F=1$ ). The FM index, designated $\beta$ in the FM nomenclature, and the AM index $M$ were determined by fits to the WMS and TTFMS line shapes recorded at different temperatures. The parameters obtained were $\beta=230$ and $M=0.045$ for WMS and $\beta=$ 1.0 and $M=0.035$ for TTFMS. With these parameter values, two curves were calculated, and they and their sum are displayed in Fig. 5, together with the potassium line experimentally recorded at $80{ }^{\circ} \mathrm{C}$.

The AM-FM index ratio $M / \beta$ is an intrinsic property of a diode laser and depends on the modulation frequency and the bias current. ${ }^{29}$ Typically, the ratio scales with the modulation frequency, and for our experiments with equal bias current for WMS and TTFMS the ratio of the AM-FM index ratio for TTFMS and WMS was 179, which is in excellent agreement with the ratio of the modulation frequencies, 181. 


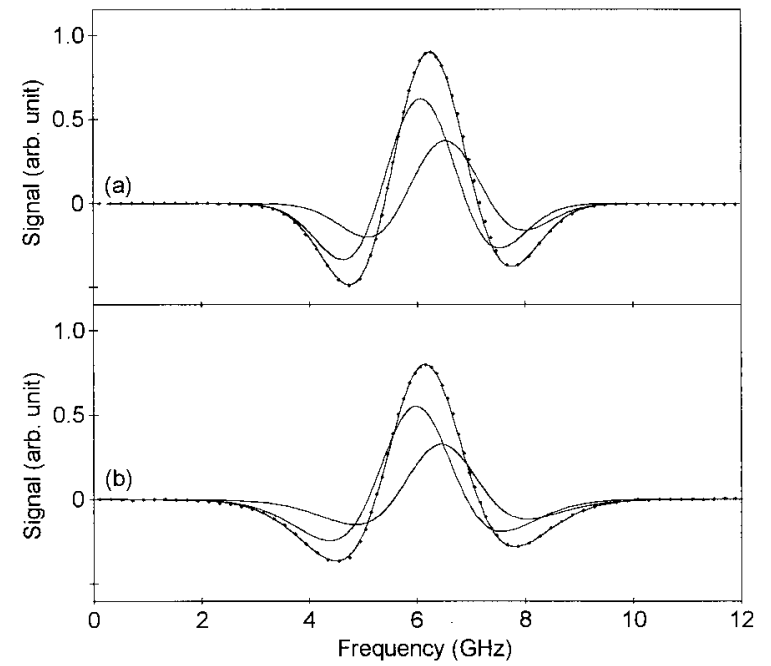

Fig. 5. Observed (symbols) and calculated (solid curves) line shapes for (a) WMS and (b) TTFMS of the potassium transition. The modulation parameters are $\beta=230$ and $M=0.045$ for WMS and $\beta=1.0$ and $M=0.035$ for TTFMS.

It can be observed from the experimental data that the signals (peak-to-peak value) in WMS are approximately 20\% higher than those of TTFMS; see Fig. $3-5$. This result agreed well with the theoretically calculated signal values that yielded a signal difference for the temperatures employed of $\sim 21 \%$ for potassium and $\sim 23 \%$ for lead. No modulation broadening is observed in either the WMS or the TTFMS line shape. This result is consistent with the experimentally applied rf powers, which we carefully adjusted for the highest signal amplitudes achievable without introducing modulation broadening. The presence of modulation broadening would make the fitting procedure more difficult and less accurate. WMS and TTFMS line shapes that were optimized only for maximum signal amplitudes showed considerable modulation broadening.

In a frequency-modulated system the power $\mathrm{SNR}_{P}$ can be expressed as ${ }^{25}$ the numerator is the time-averaged mean-squared detector current. $e$ is the charge of the electron, $\eta$ is the detector quantum efficiency, $h$ is Planck's constant, $v$ is the transition frequency, $P_{0}$ is the laser power, $Q(\alpha, \varphi)$ is a signal that is due to absorption $\alpha$ and dispersion $\varphi, M$ is the $\mathrm{AM}$ index, $N$ is the number of modulating tones, $\Delta f$ is the detection bandwidth, $k$ is Boltzmann's constant, $T$ is the absolute temperature of the detector, $R_{L}$ is the resistance of the detection system, $R(M)$ is the RAM function, $\sigma_{p}$ is the standard deviation of the laser power within the noise equivalent bandwidth of the detector system, $f$ is the detection frequency, and $\sigma_{\mathrm{ex}}$ is a systemdependent constant that is defined as the laser power fluctuations at $1-\mathrm{Hz}$ bandwidth at $1-\mathrm{Hz}$ frequency. The value of the exponent $b$ is typically 1.0 but can range from 0.8 to 1.5 .

The absorption- and dispersion-related line-shape function $Q(\alpha, \varphi)$, which depends on $M, \beta$ and $\psi$, is calculated as the peak-to-peak value of the absorption component of the WMS and TTFMS line shapes because the dispersion component vanishes as a result of our choice of detection phase. The RAM function describes the nonzero signal that is detected even in the absence of absorption and is due to the AM. The reason for employing second-harmonic detection instead of first-harmonic detection in WMS is that the influence of RAM will be decreased. For WMS and second-harmonic detection, $R(M)=1 / 4 M^{2}$ $\cos (2 \psi+\pi)$; for TTFMS, $R(M)=M^{2}$. The total power impinging upon the detector is $P_{0}=3 \mathrm{~mW}$, and the parameters for detector and detection electronics in our setup are $\eta=0.37$ and $R_{L}=50 \Omega$. From direct absorption measurements, a value of $1 \times 10^{-3}$ $P_{0}$ for the parameter $\sigma_{p}$ was deduced. The only adjustable parameter in the SNR calculations is $\sigma_{\text {ex }}$, and its value is estimated to be $4.5 \times 10^{-4} P_{0}$. These values of $\sigma_{p}$ and $\sigma_{\text {ex }}$ are approximately 1 order of magnitude higher than typical values for diode lasers used for FM in the near infrared and the infrared. ${ }^{10,14}$ We attribute the difference to the multimode behavior of our blue diode laser.

$$
\begin{aligned}
\mathrm{SNR}_{P} & =\frac{\left\langle i_{s}(t)\right\rangle^{2}}{i_{\mathrm{sn}}^{2}+i_{\mathrm{th}}^{2}+i_{\mathrm{RAM}}^{2}+i_{\mathrm{ex}}^{2}} \\
& =\frac{\left(\frac{e \eta}{h v}\right)^{2} 2\left\langle P_{0}\right\rangle^{2}|Q(\alpha, \varphi)|^{2}}{2 \frac{e^{2} \eta}{h v}\left\langle P_{0}\right\rangle\left(1+\frac{M^{2}}{2}\right)^{N} \Delta f+\frac{4 k T}{R_{L}} \Delta f+\left(\frac{e \eta}{h v}\right)^{2} 2 R(M) \sigma_{p}^{2}+\left(\frac{e \eta}{h v}\right)^{2} \frac{\Delta f}{f^{b}} \sigma_{\mathrm{ex}}^{2}},
\end{aligned}
$$

where the terms in the two expressions are in the same order and where the mean-squared noise currents in the denominator (from left to right) are related to laser-induced detector shot noise, detector and amplifier thermal noise, AM-induced (RAM) noise, and laser source or excess noise and the term in
The experimental SNR is calculated as the ratio of the line-shape peak-to-peak value to the noise rms value. Inasmuch as we measure voltage and not power, the experimental SNR is related to the theoretical voltage $\mathrm{SNR}_{V}$, which is given by $\mathrm{SNR}_{V}=$ $\left(\mathrm{SNR}_{P}\right)^{1 / 2}$. In Fig. 6 the experimental SNR's for 


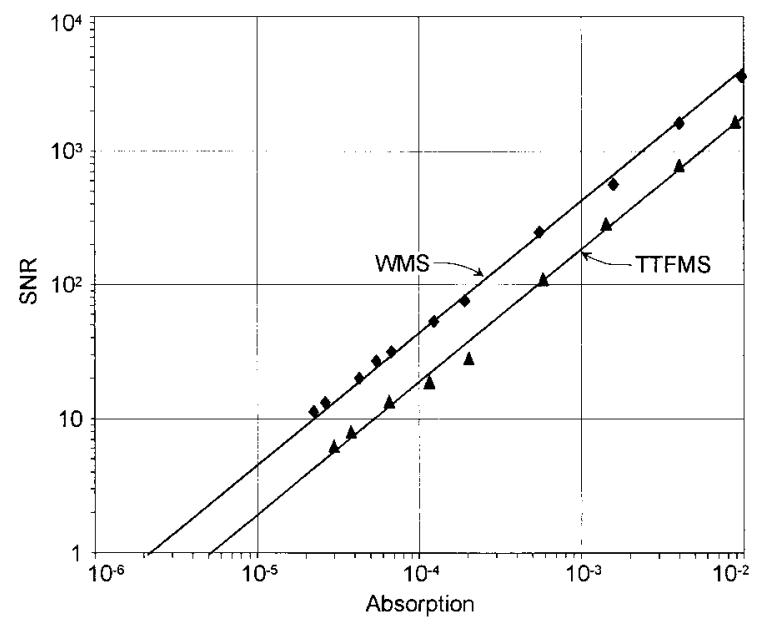

Fig. 6. Experimentally deduced SNR's for WMS (diamonds) and TTFMS (triangles) for potassium together with theoretical curves calculated from Eq. (1) and the parameter values mentioned in the text.

WMS and TTFMS are presented, together with the theoretical SNR curves. Clearly, absorption signals for less than the smallest experimentally recorded absorption of $2 \times 10^{-5}$ (at $\sim 20^{\circ} \mathrm{C}$ ) are detectable, but with our current setup it is not possible to cool the gas cell and record signals below room temperature. The sensitivity limits that can be deduced are $2 \times$ $10^{-6}$ for WMS and $5 \times 10^{-6}$ for TTFMS. In the lead experiments we were able to record WMS and TTFMS signals down to a SNR of 1 , which occurs at temperatures of $\sim 500{ }^{\circ} \mathrm{C}$ and corresponds to an absorption of $5 \times 10^{-5}$. Thus the interference fringes and the mode jump limit the detection sensitivity by $\sim 1$ order of magnitude.

There are two reasons for the approximately twotimes better SNR in WMS than in TTFMS. The first is the higher signal amplitude in WMS, and the second is the higher RAM noise level in TTFMS, as expected from the different RAM dependencies given by the RAM function. All other noise sources have the same amplitude in WMS and TTFMS. The ratio of the shot noise, the thermal noise, the laser excess noise, the TTFMS RAM noise, and the WMS RAM noise is 1:1.7:0.7:5.4:2.2. These numbers clearly show that TTFMS is essentially limited by RAM noise, whereas the sensitivity in WMS is set by both RAM and thermal noise. We also note that the laser excess noise is below the shot noise and that the main purpose for employing FM is achieved. RAM noise and, as a consequence of the technique, laser excess noise can be greatly reduced by the use of balanced homodyne detection. 13,15,21 However, the power transmitted through the absorbing medium is halved and the thermal noise is doubled in this technique. Theoretical calculations of the SNR's in balanced homodyne detection, with the same parameter values as above, show that the SNR's for TTFMS are almost the same but that the SNR's for WMS are lower, as we also verified experimentally. The results ob- tained in the present study are broadly in line with those obtained in previous comparisons of the sensitivity of various modulation schemes in which infrared lead-salt diode lasers ${ }^{10}$ and near-infrared GaAlAs diode lasers ${ }^{11}$ were used.

A possible extension of the present study would be to monitor $\mathrm{NO}_{2}$, which has strong absorption bands in the wavelength region about $400 \mathrm{~nm}$. At atmospheric pressure these transitions are much broader than for potassium and lead and might also partially overlap. Such a study would require continuous scans of the order of $50 \mathrm{GHz}$, which at present are not feasible with the diode laser used. One should also note that the multimode behavior of the diode laser does not influence the spectral appearance for free atoms with isolated spectral features as in our case, because only one of the oscillating modes interacts with the atoms. However, for molecules with a multitude of close-lying lines, multimode behavior is, of course, unacceptable. As the blue diode laser used is a prototype laser, we believe that later versions will be better suited for spectroscopy, i.e., will operate in a single mode. One can use an external-feedback cavity to ensure single-mode operation and large frequency-tuning ranges, but doing so will limit the high-FM capabilities and thus the detection sensitivity.

\section{Conclusions}

We performed FM spectroscopy on potassium and lead, using a blue diode laser. We made highly sensitive absorption measurements in this new and interesting diode-laser wavelength region, employing WMS and TTFMS. In fits to experimental data the modulation parameters for the blue diode laser used in our experimental setup were determined. We have shown that experimentally deduced SNR's are in good agreement with theoretically calculated SNR's. Additionally, various noise sources were examined, and we have concluded that TTFMS is dominated by RAM noise, whereas WMS is limited by both RAM and thermal noise. A minimum detectable absorption of $2 \times 10^{-6}$ for WMS and $5 \times 10^{-6}$ for TTFMS in a $120-\mathrm{Hz}$ detection bandwidth was observed. By sum-frequency mixing blue and red diode lasers, even shorter emission wavelengths can be achieved, further extending the possibilities for sensitive absorption spectroscopy of many atomic and molecular transitions. ${ }^{30}$

This study was supported by the Swedish Research Council for Engineering Sciences (TFR) and the Knut and Alice Wallenberg Foundation. J. Alnis thanks the Swedish Institute for a stipend supporting his stay in Sweden.

\section{References}

1. P. Werle, R. Mücke, F. D. Amato, and T. Lancia, "Nearinfrared trace-gas sensor based on room-temperature diode lasers," Appl. Phys. B 67, 307-315 (1998).

2. G. Modugno, C. Corsi, M. Gabrysch, and M. Inguscio, "Detection of $\mathrm{H}_{2} \mathrm{~S}$ at ppm level using a telecommunication diode laser," Opt. Commun. 145, 76-80 (1998). 
3. H. Riris, C. B. Carlisle, D. F. McMillen, and D. E. Cooper, "Explosives detection with a frequency modulation spectrometer," Appl. Opt. 35, 4694-4704 (1996).

4. J. A. Silver, D. J. Kane, and P. S. Greenberg, "Quantitative species measurements in microgravity flames with near-IR diode lasers," Appl. Opt. 34, 2787-2801 (1995).

5. P. Kauranen, H. M. Hertz, and S. Svanberg, "Tomographic imaging of fluid flows by the use of two-tone frequencymodulation spectroscopy," Opt. Lett. 19, 1489-1491 (1994).

6. P. Kauranen, I. Harwigsson, and B. Jönsson, "Relative vapor pressure measurements using a frequency-modulated tunable diode laser, a tool for water activity determination in solutions," J. Phys. Chem. 98, 1411-1415 (1994).

7. E. G. Moses and C. L. Tang, "High sensitivity laser wavelength modulation spectroscopy," Opt. Lett. 1, 115-117 (1977).

8. G. C. Bjorklund, "Frequency-modulation spectroscopy: a new method for measuring weak absorptions and dispersions," Opt. Lett. 5, 15-17 (1980).

9. G. R. Janik, C. B. Carlisle, and T. F. Gallagher, "Two-tone frequency-modulation spectroscopy," J. Opt. Soc. Am. B 3, 1070-1074 (1986).

10. D. S. Bomse, A. C. Stanton, and J. A. Silver, "Frequency modulation and wavelength modulation spectroscopies: comparison of experimental methods using a lead-salt diode laser," Appl. Opt. 31, 718-731 (1992).

11. F. S. Pavone and M. Inguscio, "Frequency- and wavelengthmodulation spectroscopies: comparison of experimental methods using and AlGaAs diode laser," Appl. Phys. B 56, 118-122 (1993).

12. D. E. Cooper, R. U. Martinelli, C. B. Carlisle, H. Riris, D. B. Bour, and R. J. Menna, "Measurement of ${ }^{12} \mathrm{CO}_{2}:{ }^{13} \mathrm{CO}_{2}$ ratios for medical diagnostics with $1.6-\mu \mathrm{m}$ distributed-feedback semiconductor diode lasers," Appl. Opt. 32, 6727-6731 (1993).

13. G. Modugno, C. Corsi, M. Gabrysch, F. Marin, and M. Inguscio, "Fundamental noise sources in a high-sensitivity two-tone frequency modulation spectrometer and detection of $\mathrm{CO}_{2}$ at 1.6 $\mu \mathrm{m}$ and $2 \mu \mathrm{m}, "$ Appl. Phys. B 67, 289-296 (1998).

14. C. B. Carlisle, D. E. Cooper, and H. Preier, "Quantum noiselimited FM spectroscopy with a lead-salt diode laser," Appl. Opt. 28, 2567-2576 (1989).

15. C. B. Carlisle and D. E. Cooper, "Tunable-diode-laser frequency-modulation spectroscopy using balanced homodyne detection," Opt. Lett. 14, 1306-1308 (1989).

16. P. Werle, F. Slemr, M. Gehrtz, and C. Bräuchle, "Quantumlimited FM-spectroscopy with a lead salt diode laser," Appl. Phys. B 49, 99-108 (1989).
17. M. Gehrtz, G. C. Bjorklund, and E. A. Whittaker, "Quantumlimited laser frequency-modulation spectroscopy,” J. Opt. Soc. Am. B 2, 1510-1526 (1985).

18. S. Nakamura and G. Fasol, The Blue Laser Diodes (SpringerVerlag, Heidelberg, 1997).

19. U. Gustafsson, J. Alnis, and S. Svanberg, "Atomic spectroscopy with violet laser diodes," Am. J. Phys. (to be published).

20. P. Kauranen and V. G. Avetisov, "Determination of absorption line parameters using two-tone frequency-modulation spectroscopy with diode lasers," Opt. Commun. 106, 213-217 (1994).

21. V. G. Avetisov and P. Kauranen, "High-resolution absorption measurements using two-tone frequency-modulation spectroscopy with diode lasers," Appl. Opt. 36, 4043-4054 (1997).

22. V. G. Avetisov and P. Kauranen, "Two-tone frequencymodulation spectroscopy for quantitative measurements of gaseous species: theoretical, numerical, and experimental investigation of line shapes," Appl. Opt. 35, 4705-4723 (1996).

23. E. Arimondo, M. Inguscio, and P. Violino, "Experimental determinations of the hyperfine structure in the alkali atoms," Rev. Mod. Phys. 49, 31-75 (1977)

24. D. E. Cooper and R. E. Warren, "Frequency modulation spectroscopy with lead-salt diode lasers: a comparison of singletone and two-tone techniques," Appl. Opt. 26, 3726-3732 (1987).

25. J. A. Silver, "Frequency-modulation spectroscopy for trace species detection: theory and comparison among experimental methods," Appl. Opt. 31, 707-717 (1992).

26. J. M. Supplee, E. A. Whittaker, and W. Lenth, "Theoretical description of frequency modulation and wavelength modulation spectroscopy," Appl. Opt. 33, 6294-6302 (1993).

27. W. Lenth, "High frequency heterodyne spectroscopy with current-modulated diode lasers," IEEE J. Quantum Electron. QE-20, 1045-1050 (1984).

28. S. Kobayashi, Y. Yamamoto, M. Ito, and T. Kimura, "Direct frequency modulation in AlGaAs semiconductor lasers," IEEE J. Quantum Electron. QE-18, 582-595 (1982).

29. M. Osinski and J. Buus, "Linewidth broadening factors in semiconductor lasers: an overview," IEEE J. Quantum Electron. QE-23, 9-29 (1987).

30. J. Alnis, U. Gustafsson, G. Somesfalean, and S. Svanberg, "Sum-frequency generation with a blue diode laser for mercury spectroscopy at $254 \mathrm{~nm}$," Appl. Phys. Lett. 76, 1234-1236 (2000). 